\title{
Effect of microwave irradiation on spin-torque-driven magnetization precession in nanopillars with magnetic perpendicular anisotropy
}

\author{
N. Reckers,,${ }^{1,}$ J. Cucchiara, ${ }^{2}$ O. Posth, ${ }^{1}$ C. Hassel,,${ }^{1}$ F. M. Römer, ${ }^{1}$ R. Narkowicz, ${ }^{3}$ R. A. Gallardo,${ }^{4}$ P. Landeros, ${ }^{4}$ H. Zähres,${ }^{1}$ \\ S. Mangin, ${ }^{2}$ J. A. Katine, ${ }^{5}$ E. E. Fullerton, ${ }^{6}$ G. Dumpich, ${ }^{1}$ R. Meckenstock, ${ }^{1}$ J. Lindner, ${ }^{1}$ and M. Farle ${ }^{1}$ \\ ${ }^{1}$ Faculty of Physics and Center for Nanointegration (CeNIDE), Lotharstr. 1, DE-47057 Duisdurg, Germany, \\ and University of Duisburg-Essen, Lotharstr. 1, DE-47057 Duisburg, Germany \\ ${ }^{2}$ Nancy-Université, Laboratoire de Physique des Matériaux, CNRS, Boîte Postal 239, FR-54506 Vandoeuvre lés Nancy, France \\ ${ }^{3}$ Department of Physics, University of Dortmund, Otto-Hahn-Str. 4, DE-44227 Dortmund, Germany \\ ${ }^{4}$ Departamento de Física, Universidad Técnica Federico Santa María, Avenida España 1680, 2390123 Valparíso, Chile \\ ${ }^{5}$ Hitachi Global Storage Technologies, Yerba Buena Road, San Jose, California 95135, USA \\ ${ }^{6}$ Center of Magnetic Recording Research and Department of Electrical and Computer Engineering, University of California, San Diego, \\ 9500 Gilman Drive, La Jolla, California 92093-0401, USA
}

(Received 10 January 2011; revised manuscript received 24 March 2011; published 25 May 2011)

\begin{abstract}
The effect of microwave irradiation on the spin-torque-driven magnetization dynamics is studied in $(\mathrm{Co} / \mathrm{Ni})$ based nanopillar spin valves with perpendicular magnetic anisotropy. For this purpose, a setup was developed to measure the ac as well as the dc resistance of the nanopillar under applied fields and injected polarized currents, while irradiating microwaves with varying frequency $(6-18 \mathrm{GHz})$ and power. We find that the microwave irradiation amplifies and maintains the precessional state of the eigenresonance within a larger field range. The experiments are discussed in comparison to micromagnetic as well as macrospin simulations utilizing the nonlinearized Landau-Lifshitz-Gilbert equation.
\end{abstract}

DOI: 10.1103/PhysRevB.83.184427

PACS number(s): 75.78.-n, 75.47.-m, 85.75.-d, 72.25.Ba

\section{INTRODUCTION}

Within nanopillar spin valves consisting of a ferromagnetic hard layer, acting as a spin polarizer, and a ferromagnetic soft (free) layer separated by a nonmagnetic spacer layer, the spintorque $^{1,2}$ effect manifests itself by magnetization switching and by steady-state precessions. ${ }^{3,4}$ Many investigations were conducted for systems with an easy axis of magnetization in the film plane that demonstrate the steady-state precession frequencies in the microwave regime, allowing the effect to be employed for microwave generation. ${ }^{5,6}$ A theory to understand microwave generation by spin-polarized currents is provided in Ref. 7 Motivated by the possibility of dc-induced microwave generation, the influence of rf excitation on systems with an easy axis of magnetization in the film plane was studied at low frequencies (below $15 \mathrm{GHz}$ ) using rf currents ${ }^{8}$ and at high frequencies $(40-60 \mathrm{GHz})$ using microwave irradiation. ${ }^{9}$

Experiments on nanopillars with perpendicular magnetic anisotropy (PMA) revealed that the current densities needed for magnetization switching are effectively reduced ${ }^{10}$ as compared to in-plane systems. While a precessional elliptical state can easily be studied for in-plane magnetized samples, it is far more complicated in the case of PMA-based systems because the circular precession of the magnetization perpendicular to the film plane does not change the giant magnetoresistance (GMR) signal. ${ }^{11}$ Consequently, in investigations of PMAswitching layers, either polarizing layers with in-plane easy magnetization ${ }^{12}$ or a third in-plane magnetized ferromagnetic layer that indirectly monitors the motion of the PMA-switching layer were used. ${ }^{11} \mathrm{~A}$ recent theoretical work discussed the influence of microwave irradiation on $(\mathrm{Co} / \mathrm{Ni})$-based systems with PMA and showed that microwave-assisted switching might be indeed beneficial. ${ }^{13}$

In the present paper, the spin-transfer-torque effect within (Co/Ni)-based systems with PMA is experimentally studied under microwave irradiation in the frequency range of 5$11 \mathrm{GHz}$, which is in the range of the magnetization eigenresonance. The investigation focuses on the effect of microwave irradiation on the precessional state of the system rather than on microwave-assisted switching. Our study provides unambiguous evidence that (i) the resonance frequency of nanopillars for arbitrary orientation of the two magnetizations, including the special case of two PMA-based materials, can be determined and (ii) the critical current density needed to excite the steady-state precession is reduced by microwave irradiation.

\section{EXPERIMENTAL DETAILS}

The layout of the experimental setup is schematically shown in Fig. 1. The ac resistance $(d V / d I)$ and the dc resistance can be measured in the presence of a magnetic field while microwaves in the gigahertz range are irradiating the sample. The setup to detect the magnetoresistance is based on a modified version of the measurement bridge described in Ref. 14. The detection limit is $\frac{\Delta R}{R}=10^{-5}$. The magnetic field can be swept up to $2 \mathrm{~T}$ and can be oriented parallel or perpendicular to the sample plane. To enable microwave broadband irradiation, a coaxial semirigid microwave cable (SRMC) with a diameter of $2 \mathrm{~mm}$ is utilized (which is described in more detail in the Appendix).

The samples used for the investigations are made of $(\mathrm{Co} / \mathrm{Ni})$-based PMA materials. The magnetically hard layer acting as a spin polarizer is given by a $(\mathrm{Co} / \mathrm{Pt})-(\mathrm{Co} / \mathrm{Ni})$ multilayer while the free (switching) layer is given by a $(\mathrm{Co} / \mathrm{Ni})$ multilayer. Both layers are separated from each other by a copper spacer layer. Details of the sample preparation have been published elsewhere. ${ }^{15-17}$ The films were patterned to form $50 \times 300 \mathrm{~nm}^{2}$ nanopillars. A total of eight devices 


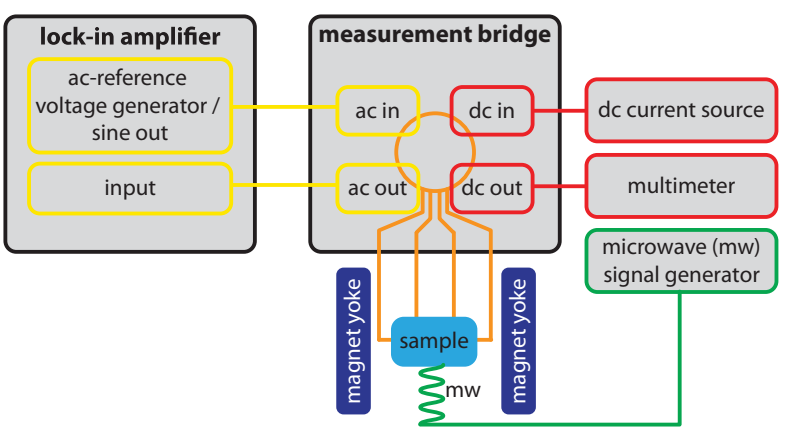

FIG. 1. (Color online) Schematic overview of the experimental setup. The connection of the different components with the measurement bridge, which is a modification of the bridge described in Ref. 14, enables measurement of the magnetoresistance. The signal detected by means of the lock-in technique relies on the use of a modulated alternating current with a dc offset. The measurement bridge is an ac low-resistance bridge only allowing the measurement of resistances up to $20 \Omega$.

were measured and showed a resistance of $3.3 \pm 0.02 \Omega$ in the parallel alignment and a GMR ratio of $\frac{\Delta R}{R}=0.25 \%$. All measurements shown here were performed at room temperature with the magnetic field applied perpendicularly to the film plane.

\section{RESULTS AND DISCUSSION}

Figure 2(a) shows the resistance change versus the magnetic field. We observed the magnetic switching of the free and fixed layer by applying a small ac current (with frequency $f=$ $997 \mathrm{~Hz}$ and amplitude $A=40 \mu \mathrm{A}$ ) without a dc current, $I_{\mathrm{dc}}$. The measurement starts in the parallel alignment of both layers at a high magnetic field (of $500 \mathrm{mT}$ ). The free layer switches from the parallel to the antiparallel state at $B= \pm 95 \mathrm{mT}$. By increasing the magnetic field the hard layer switches from antiparallel to parallel alignment at $B= \pm 410 \mathrm{mT}$. Between -500 and $500 \mathrm{mT}$ we find an overall symmetric curve with respect to the zero field. The observed response can be explained by the GMR effect. Without any injected dc current the switching field of the hard $(\mathrm{Co} / \mathrm{Pt})-(\mathrm{Co} / \mathrm{Ni})$ layer is about $400 \mathrm{mT}$. After saturation, the maximum magnetic field is limited to $250 \mathrm{mT}$ to avoid any magnetization change in the hard layer when measuring the minor loop.

Figure 2(b) shows the resistance change as a function of $I_{\mathrm{dc}}$. The measurement starts in the parallel alignment of the magnetization at $I_{\mathrm{dc}}=9 \mathrm{~mA}$. At a critical dc current, $I_{C}^{-}=-5.2 \mathrm{~mA}$, the configuration switches from parallel to antiparallel alignment and at $I_{C}^{+}=2.6 \mathrm{~mA}$ the configuration switches back to the parallel alignment. Considering the elliptical shape of the sample, we calculate the critical current densities $J_{C}^{-} \approx 1.10 \times 10^{7} \mathrm{~A} / \mathrm{cm}^{2}\left(I_{C}^{-}=-5.2 \mathrm{~mA}\right)$ and $J_{C}^{+} \approx 5.52 \times 10^{6} \mathrm{~A} / \mathrm{cm}^{2}\left(I_{C}^{+}=2.6 \mathrm{~mA}\right)$. The parabolic shape is due to Joule heating. To avoid this parabolic background for all measurements shown in the following, the external magnetic field was varied at a constant dc current, $I_{\mathrm{dc}}$. The shifted zero point of the parabola is due to the Peltier effect. ${ }^{18}$ The measurement in Fig. 2(b) was performed at the remanence field of the magnet (which is $3 \mathrm{mT}$ ).
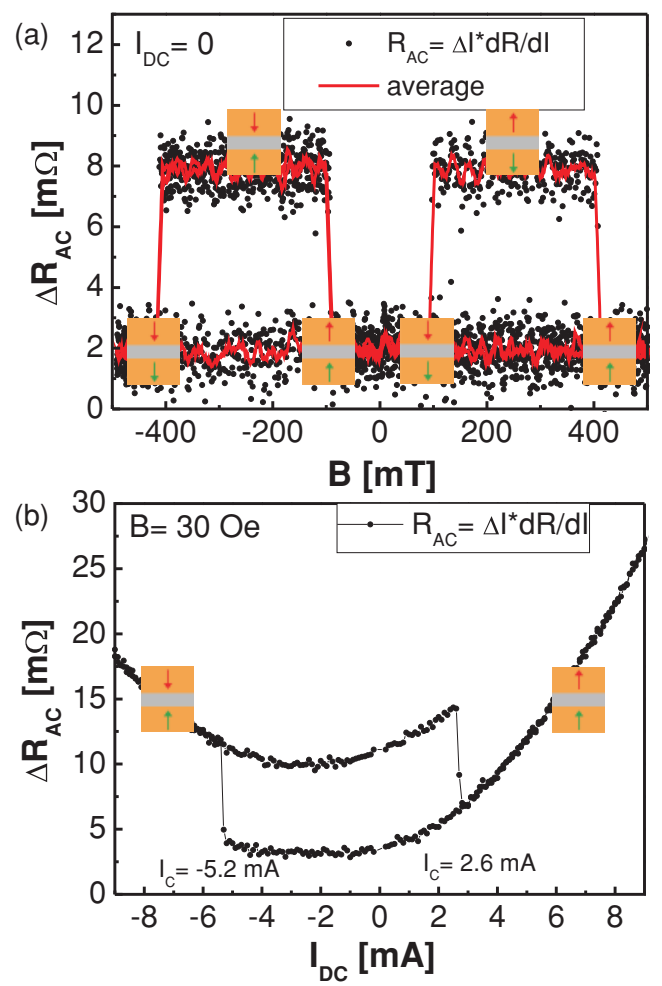

FIG. 2. (Color online) (a) The ac resistance change as a function of the perpendicular magnetic field. (b) The ac resistance change as a function of the dc current.

Figure 3 shows the evolution of the ac resistance change, $\Delta R_{\mathrm{ac}}$, as a function of the magnetic field for several injected currents. $I_{\mathrm{dc}}$ is defined as positive if the electrons flow from the hard $(\mathrm{Co} / \mathrm{Pt})-(\mathrm{Co} / \mathrm{Ni})$ layer (polarizer) to the free $(\mathrm{Co} / \mathrm{Ni})$ layer. Two types of ac resistance curves were observed.

For $I_{\mathrm{dc}}<9 \mathrm{~mA}$ a hysteretic behavior is observed, as shown in Figs. 3(a) and 3(b). We note that for $I_{\mathrm{dc}}=0$ a square hysteresis loop shifted toward negative magnetic fields by about $30 \mathrm{mT}$ due to the dipolar field generated by the hard layer ${ }^{19}$ is observed (although it is not shown in this work). The coercivity at $I_{\mathrm{dc}}=0$ is $95 \mathrm{mT}$ [see Fig. 2(a)].

As $I_{\mathrm{dc}}$ increases, the coercivity decreases and the hysteresis loop shifts toward larger negative fields as shown in Figs. 3(a) and 3(b) for $I_{\mathrm{dc}}=6 \mathrm{~mA}$. This is consistent with the analytical results of Fig. 6(a) and it is due to the spin-transfer torque as explained in detail in Ref. 6. The switching fields are now -117 and $-70 \mathrm{mT}$, respectively.

For $I_{\mathrm{dc}}>9 \mathrm{~mA}$ a peak in the ac resistance is observed as shown in Fig. 3(c). The maximum of the peak occurs at a magnetic field of $-190 \mathrm{mT}$. Note that for $I_{\mathrm{dc}}>9 \mathrm{~mA}$ a peak in the ac magnetoresistance can also be observed. By analyzing the dc resistance changes, $R_{\mathrm{dc}}$ [see Figs. 3(b) and 3(d)], one can conclude that the process of magnetization movement is irreversible for low dc currents and reversible for larger ones (for which the peak is observed in the ac resistance change). For a dc current of $6 \mathrm{~mA}$ a hysteresis is observed, just like for the ac resistance change, implying an irreversible process. This irreversibility is given by the switching of the magnetization 

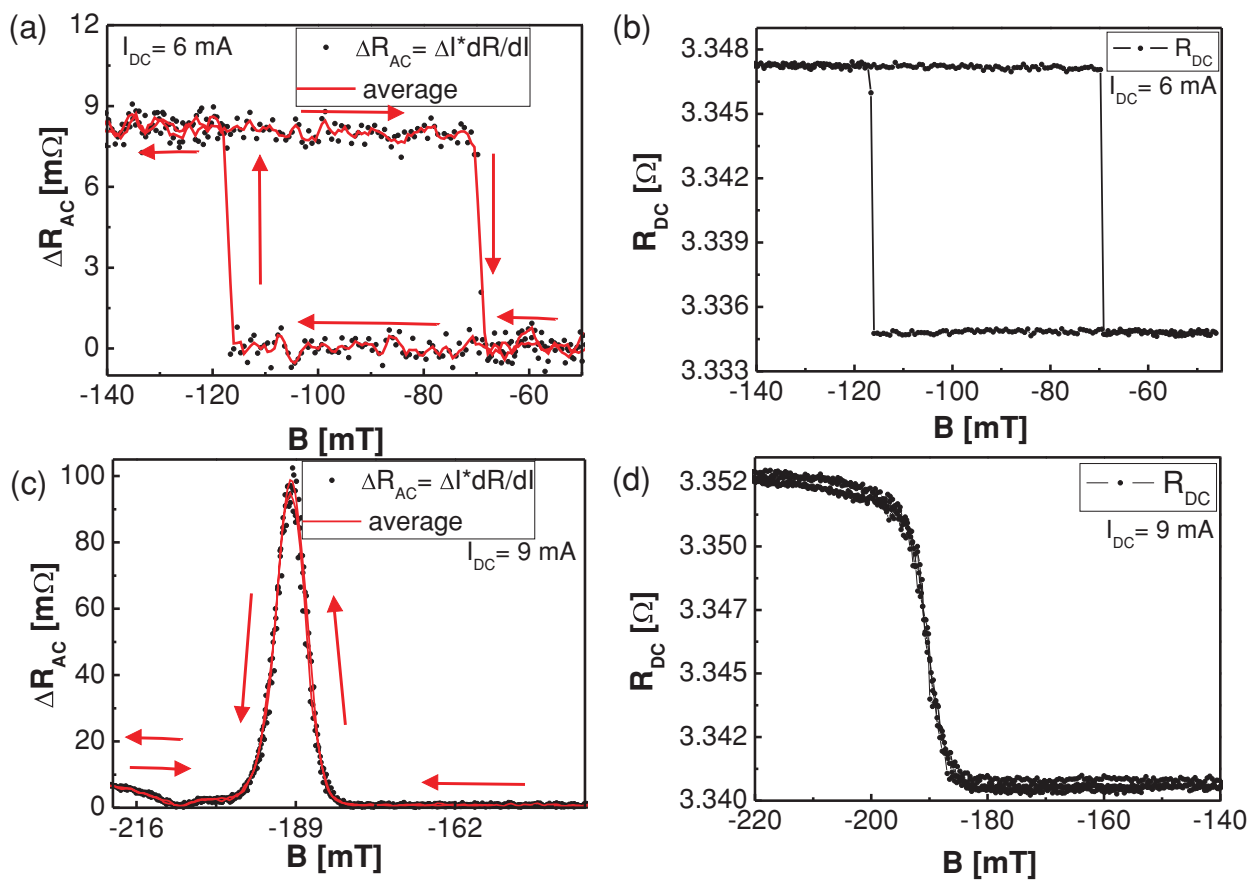

FIG. 3. (Color online) The ac resistance change (left column) and the dc resistance (right column) as a function of the perpendicular magnetic field for different dc currents, $I_{\mathrm{dc}}$.

between parallel and antiparallel alignment relative to the magnetization of the polarizer. At $I_{\mathrm{dc}}=9 \mathrm{~mA}$, however, the $\mathrm{dc}$ resistance change is nonhysteretic, clearly indicating a reversible change of the magnetization direction. In this case, $\Delta R_{\mathrm{ac}}$ is just given by the derivative of the dc resistance change producing the peaklike behavior. As discussed in detail below and observed by other authors before, ${ }^{5,6}$ the reversible change is given by a steady-state precession of the magnetization and is caused by the competition between the external field, which favors an antiparallel alignment of the magnetizations, and the spin-torque, which supports the parallel orientation of the two magnetizations. The effect of the microwave irradiation in the gigahertz range on $\Delta R_{\mathrm{ac}}$ is shown in Fig. 4(a). The ac resistance curves are obtained for a constant dc current of $I_{\mathrm{dc}}=10 \mathrm{~mA}$ while sweeping the magnetic field up to $-250 \mathrm{mT}$ without irradiating the device. It is compared to the measurement performed under identical conditions but with microwave irradiation of $7.7 \mathrm{GHz}$. When the sample is not irradiated, a peak in $\Delta R_{\mathrm{ac}}$ is observed at $B=-188$ mT. When the sample is irradiated at $7.7 \mathrm{GHz}$, the peak is only slightly shifted and becomes broader and higher. The increasing amplitude of the peak is a hint that the microwave irradiation enhances the magnetization precessional state. The fact that the peak is broader under microwave irradiation shows that a precession can be excited for a wider field range. This is comparable to the behavior also observed in the mutual phase locking of two spin-torque oscillators. ${ }^{20,21}$

Figure 4(b) shows the evolution of the ac resistance, $\Delta R_{\mathrm{ac}}$, for a fixed field of $-190 \mathrm{mT}$ and $I_{\mathrm{dc}}=10 \mathrm{~mA}$ as a function of the frequency tuned from 5 to $11 \mathrm{GHz}$. A maximum is measured at $7.75 \mathrm{GHz}$ [which is very close to the zerofrequency peak, the blue line in Fig. 4(a)]. The signal under microwave irradiation at $f=7.75 \mathrm{GHz}$ is strongly enhanced. Two smaller modes are observed at $f=9.4$ and $10.3 \mathrm{GHz}$. A clear enhancement of the ac resistance signal is observed for frequencies from 7 to $9 \mathrm{GHz}$. The fact that several peaks appear in the frequency spectrum is due to several oscillation modes; this is supported by the micromagnetic simulations discussed below.

The device shows a situation where the almost closed hysteresis is overlapped by the precession peak at $9 \mathrm{~mA}$, as shown in Fig. 4(c), with additional microwave irradiation at a frequency of $7.9 \mathrm{GHz}$. This shows that the microwave irradiation gives rise to magnetization precession and that the critical current needed to create magnetization oscillation can be reduced by applying a microwave field tuned to the resonance frequency.

\section{THEORETICAL DESCRIPTION}

\section{A. Micromagnetic simulations of the precessional states}

To obtain a better understanding of the precessional state we performed micromagnetic simulations using the objectoriented micromagnetic framework (OOMMF) ${ }^{22}$ code. The normal modes of the elliptically shaped pillar were calculated by using the same magnetic field of $-190 \mathrm{mT}$ perpendicular to the layers ( $z$ direction) as in the experiment [see Fig. 4(b)]. In addition, a small magnetic field of $1 \mathrm{mT}$ in the $x$ direction (i.e., in the layer plane along the long axis of the elliptical pillar) is applied to generate a small deviation of the magnetization away from the film normal. After saturation, the small field is removed and the time evolution of the magnetization precession is calculated. The Fourier transformation directly 

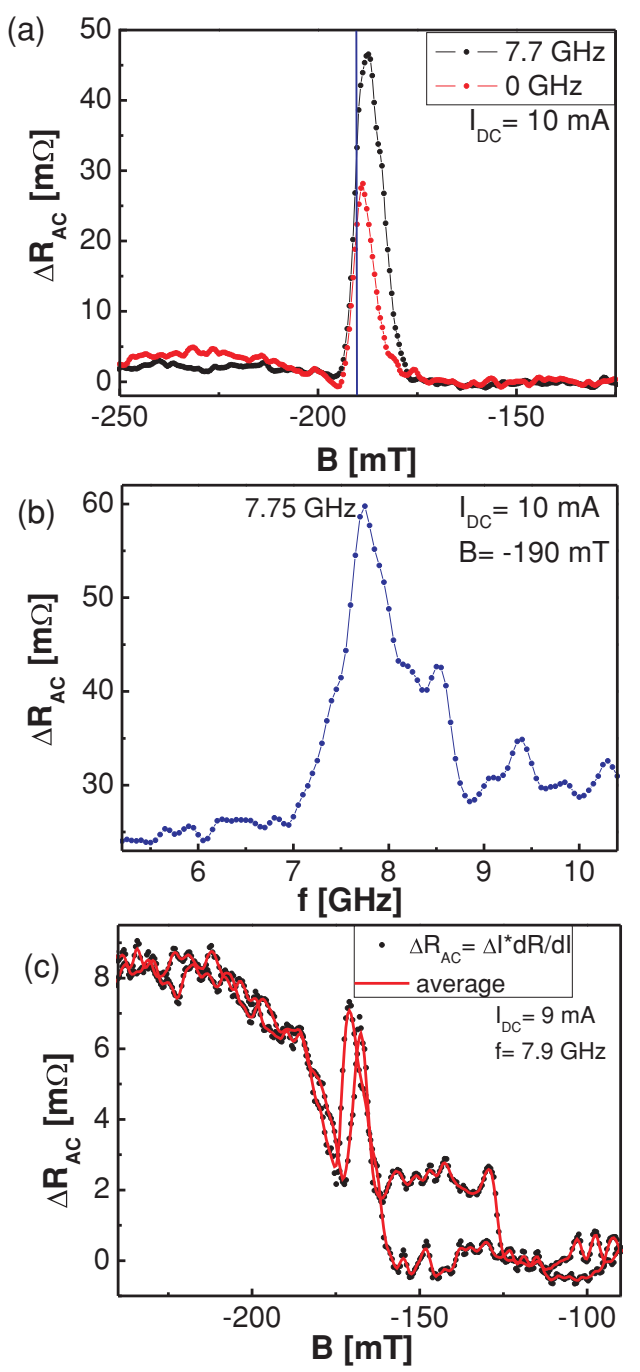

FIG. 4. (Color online) (a) The ac resistance change over the magnetic field for $I_{\mathrm{dc}}=10 \mathrm{~mA}$ without microwave irradiation (red line) and with a microwave at $f=7.7 \mathrm{GHz}$ (blue line). (b) The frequency-dependent measurement of the resistance change. (c) The ac resistance change over the magnetic field for a dc current of $9 \mathrm{~mA}$ and $f=7.9 \mathrm{GHz}$.

yields the normal, calculated modes as a function of frequency. Note that no spin-torque term has been included in the calculation.

Figure 5 shows the signal amplitude as a function of the microwave frequency. One clearly observes a main mode in the spectrum and two smaller modes located at higher frequencies. (Note that the amplitude of the smaller modes was multiplied by a factor of 10.) For every mode, we have plotted snapshots of the magnetization state within the pillar at the respective frequency maxima of the amplitude [see Figs. 5 (a)-5(c)]. The different colors visualize these deviations from the equilibrium direction ( $z$ direction); red indicates a dynamic component along the $x$ direction in the film plane and blue indicates a deviation along the $-x$ direction. Blue and red spots, therefore, show spins precessing $180^{\circ}$ out of phase.

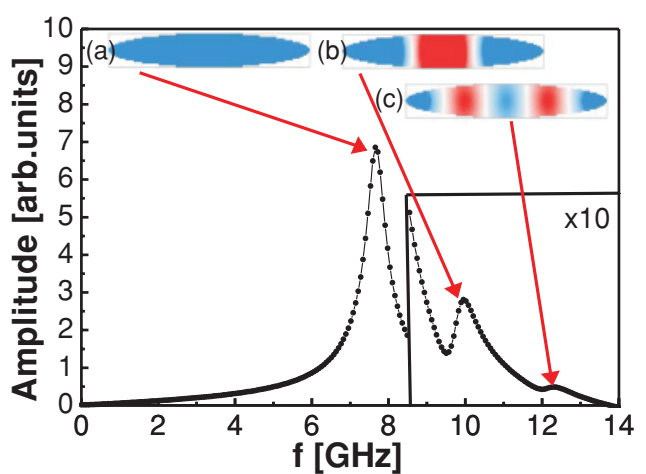

FIG. 5. (Color online) OOMMF calculation of the amplitude as a function of frequency. Visualizations of the different magnetization states at the maxima of the amplitude reveal (a) a uniform mode and (b) and (c) spin-wave modes (for details see text).

For the main mode [see Fig. 5(a)] all magnetic moments precess in phase. The snapshot of the first smaller mode [see Fig. 5(b)] shows two nodes, implying that there are phase shifts of the precession along the $x$ direction. Thus the mode can be identified with a so-called forward-volume mode, for which the propagation vector, $k$, is oriented in the plane along the long axis of the elliptical pillar while the equilibrium magnetization is aligned out of plane along the film normal. ${ }^{23}$ The name "forward" mode stems from the fact that for such modes the group velocity is positive (in contrast to the so-called backward-volume modes; see Ref. 23 for details). The snapshot of the second smaller mode [see Fig. 5(c)] exhibits four nodes.

The calculated spectrum corresponds to the experimental one of Fig. 4(b) when the following parameters are used: uniaxial out-of-plane anisotropy $K_{2 \perp}=2.23 \times 10^{5} \mathrm{~J} / \mathrm{m}^{3}$, saturation magnetization $M_{s}=617 \times 10^{3} \mathrm{~A} / \mathrm{m}$, a gyromagnetic ratio of $\gamma=175.87 \mathrm{GHz} / \mathrm{T}$, and a Gilbert damping parameter $\alpha=0.1$ (taken from Ref. 13). The uniform mode appears at the same frequency as in the experiment $(f=\omega / 2 \pi=7.7 \mathrm{GHz})$ and the spin-wave excitations appear at higher frequencies. Their frequencies are 10.0 and $12.3 \mathrm{GHz}$.

\section{B. Macrospin dynamics}

To obtain a better understanding of the dynamics of the device we have modeled its current-field phase diagram, obtained by solving the nonlinearized LandauLifshitz-Gilbert (LLG) equation with the spin-torque contribution, ${ }^{2}$

$$
\begin{aligned}
\frac{d \vec{M}}{d t}= & -\gamma\left(\vec{M} \times \vec{B}_{\mathrm{eff}}\right)+\frac{\alpha}{M_{s}} \vec{M} \times \frac{\vec{M}}{d t} \\
& -\frac{\gamma \beta}{M_{s}} \vec{M} \times(\vec{M} \times \vec{p}) .
\end{aligned}
$$

Here $M_{s}$ is the saturation magnetization, $\gamma=g \mu_{B} / \hbar$ is the gyromagnetic ratio (where $g$ is the $g$ factor), and $\alpha$ is the Gilbert-damping parameter. We chose a coordinate system such that the $z$ axis of the Cartesian coordinate system coincides with the direction normal to the layers. The first term on the right-hand side describes the precession of the 

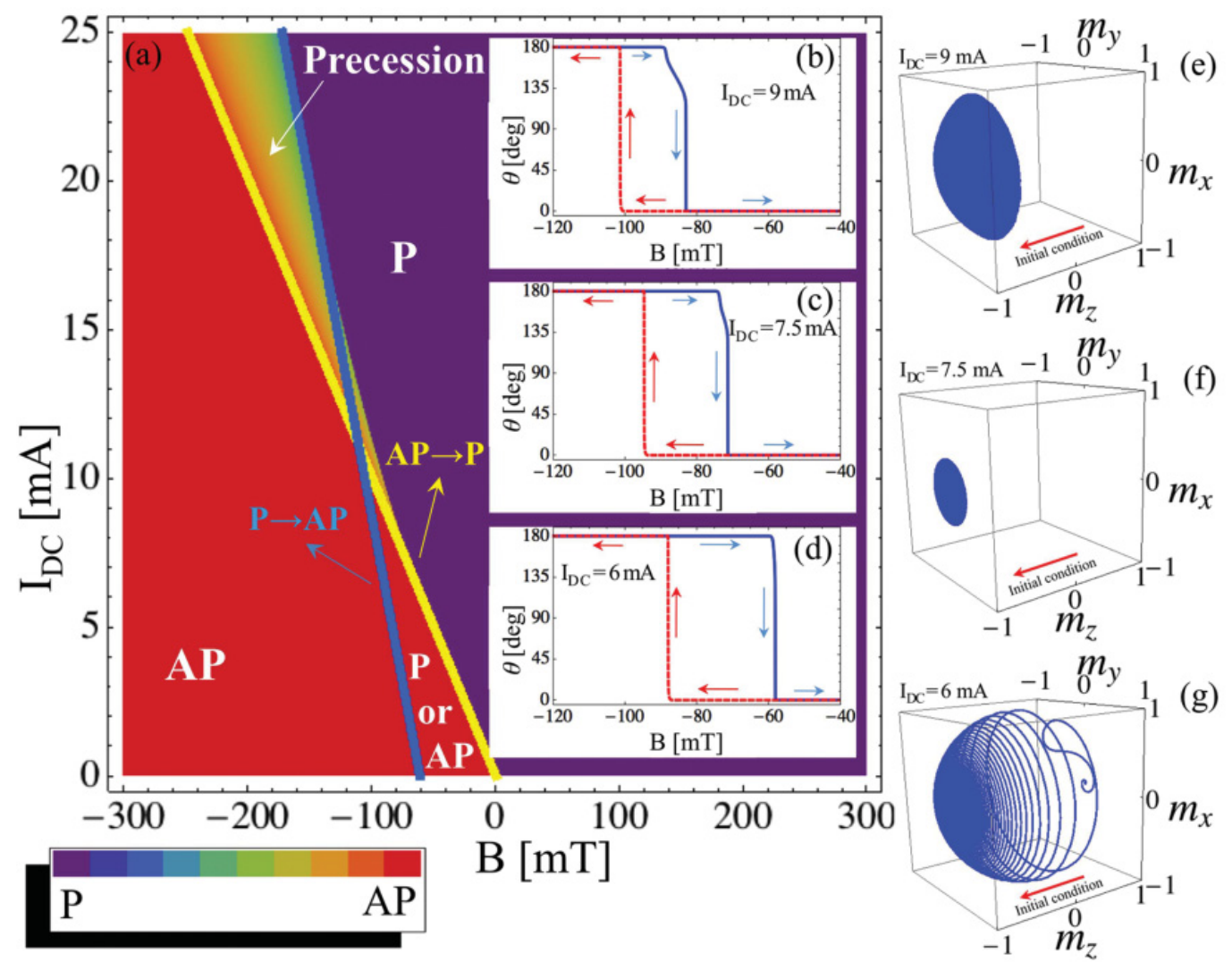

FIG. 6. (Color online) (a) Phase diagram showing the dc current, $I_{\mathrm{dc}}$, vs an external magnetic field $B$ for the stable parallel alignment (P) of the $(\mathrm{Co} / \mathrm{Ni})$ layer and polarizer, the stable antiparallel alignment (AP), the steady-state precession, and a bistable state. The latter describes the hysteretic switching between $\mathrm{P}$ and AP alignment. (b)-(d) The field dependence of the out-of-plane equilibrium angle, $\theta$, of the (Co/Ni) magnetization for different (fixed) values of the dc current: (e) $I_{\mathrm{dc}}=9 \mathrm{~mA}$, (f) $I_{\mathrm{dc}}=7.5 \mathrm{~mA}$, and (g) $I_{\mathrm{dc}}=6 \mathrm{~mA}$. The solid and dashed lines indicate the direction of the external magnetic field sweep (see text). (e) and (f) The trajectory of the $(\mathrm{Co} / \mathrm{Ni})$ magnetization for the three dc current values. The solid and dashed lines indicate the direction of the external magnetic field sweep as indicated by the arrows. The external field values for the calculation are $B=-83,-73$, and $-54 \mathrm{mT}$, respectively. The $z$ direction was chosen to be aligned parallel to the film normal.

macrospin driven by the effective field, $B_{\text {eff. The latter is }}$ given by the external dc magnetic field, $B$, including the field produced by the polarizer stray field (measured at $30 \mathrm{mT}$ ) and the effective magnetization of the sample, $M_{\mathrm{eff}}=$ $2 K_{2 \perp} / M_{s}-N \mu_{0} M_{s}$. While the shape anisotropy, $N \mu_{0} M_{s}$, always favors an easy axis in the plane of the pillar, the intrinsic out-of-plane anisotropy field, $2 K_{2 \perp} / M_{s}$, with positive $K_{2 \perp}$ overcompensates the shape anisotropy and stabilizes the out-of-plane easy axis of magnetization observed in $(\mathrm{Co} / \mathrm{Ni})$ multilayers. Details of the interplay between the two contributions to $M_{\text {eff }}$ and their dependence on the growth conditions in thin film samples have been discussed in Ref. 24. For the calculation, the same parameters as for the OOMMF simulation were chosen, only $K_{2 \perp}=2.35 \times 10^{5} \mathrm{~J} / \mathrm{m}^{3}$ was taken to be $5 \%$ larger than the one used in the OOMMF calculation. Note that the small lateral dimensions of the elliptical $(\mathrm{Co} / \mathrm{Ni})$ layer lead to a reduction of the demagnetizing factor, $N_{z} \approx 0.94$ (see Ref. 25), as compared to a thin film.

The second term on the right-hand side of Eq. (1) is the Gilbert-damping term with the phenomenological damping parameter $\alpha$. The last term describes the contribution of the spin torque as proposed by Slonczewski ${ }^{2}$ with

$$
\beta=\frac{\hbar g_{P} I_{\mathrm{dc}}}{2|e| M_{s} d V},
$$

where $d$ is the film thickness, $J$ is the current density defined as positive for the case when the electrons flow from the polarizer to the $(\mathrm{Co} / \mathrm{Ni})$ layer, and $g_{P}=1 /\left(-4+\frac{\left(3+m_{z}\right)(1+P)^{3}}{4 P^{3 / 2}}\right)$ is the polarization function. It should be noted that the Slonszweski term can have two effects: it may lead to additional damping of the system or to a precessional contribution that drives the magnetization, that is, $\beta>0$ or $\beta<0$. This is shown, e.g., in Ref. 26.

After solving Eq. (1), we get the following set of nonlinear equations that describe the time evolution of the components of the magnetization (unit) vector, $m_{i}=M_{i} / M_{s}$ :

$$
\begin{aligned}
& \dot{m}_{x}=\alpha\left(m_{y} \dot{m}_{z}-m_{z} \dot{m}_{y}\right)-\gamma m_{y}\left(B+M_{\mathrm{eff}} m_{z}\right)-\gamma \beta m_{x} m_{z}, \\
& \dot{m}_{y}=\alpha\left(m_{z} \dot{m}_{x}-m_{x} \dot{m}_{z}\right)+\gamma m_{x}\left(B+M_{\mathrm{eff}} m_{z}\right)-\gamma \beta m_{y} m_{z}, \\
& \dot{m}_{z}=\alpha\left(m_{x} \dot{m}_{y}-m_{y} \dot{m}_{x}\right)-\gamma \beta\left(m_{z}^{2}-1\right) .
\end{aligned}
$$


Figure 6(a) shows the resulting phase diagram constructed by solving the above equations for different current and external magnetic field combinations. The parameters needed for the calculation were the same as in the OOMMF simulation discussed above. The diagram is plotted for positive current values and positive and negative field values. The results for a given current-field combination are also shown in Figs. 6(e)-6(g). To obtain the plots, we have computed three magnetization components, $m_{i}$, that describe the trajectory of the magnetization vector from the above equations. Note that the $z$ direction was chosen to coincide with the direction perpendicular to the layers in the pillar. Consequently, the $x$ and $y$ components are oriented within the plane of the $(\mathrm{Co} / \mathrm{Ni})$ film. For $I_{\mathrm{dc}}=6 \mathrm{~mA}$ [see Fig. $\left.6(\mathrm{~g})\right]$ and $B=-54 \mathrm{mT}$ the $(\mathrm{Co} / \mathrm{Ni})$ magnetization starts to precess from its initial orientation parallel to the polarizer (i.e., parallel to the $-z$ direction) toward a stable antiparallel alignment. At this current value one obtains current-induced switching of the (Co/Ni) magnetization. In contrast, Figs. 6(e) and 6(f) show the situation for the higher current values of $I=7.5 \mathrm{~mA}$ and $B=-73 \mathrm{mT}$ and $I=9 \mathrm{~mA}$ and $B=-83 \mathrm{mT}$. In this case, a steady-state precession is excited. The opening angle depends on the magnitude of the magnetic field and the dc current.

The complete phase diagram is obtained by calculating the trajectories for different current-field values. Stable parallel and antiparallel orientation of the $(\mathrm{Co} / \mathrm{Ni})$ layer and the polarizer are denoted by $\mathrm{P}$ and AP, respectively. In the case of hysteretic switching, one also obtains current-field values for which both orientations are stable depending on the history of the system. The boundary between this bistable area and the area with either a stable parallel or an antiparallel alignment is given by the blue and yellow lines in the phase diagram, which can be calculated from Eq. (3) (with a detailed calculation being given in Appendix B). When sweeping the external magnetic field, $B$, at $I_{\mathrm{dc}}=0$, one obtains a classical field-driven hysteresis. The two coercive fields are shifted by the stray field of the polarizer (which is about $30 \mathrm{mT}$ ) toward negative field values. As can be seen in the phase diagram, this leads to coercive fields of about -80 and $+20 \mathrm{mT}$. Upon increasing the dc current the field swept hysteresis is shifted even more toward negative fields and the coercivity is decreased. This behavior is in accordance with the experimental observation shown in Figs. 3(a) and 3(b).

At $I_{\mathrm{dc}}=7$ to $8 \mathrm{~mA}$ the calculation predicts a steady-state precession of the $(\mathrm{Co} / \mathrm{Ni})$ magnetization around the film normal, as seen in Figs. 3(d) and in 3(c). The region in phase space for which a steady-state precession at negative external magnetic fields becomes possible increases for higher dc currents.

Furthermore, Figs. 6(b)-6(d) show-for the same current values as used for calculating the trajectories - the field dependence of the polar out-of-plane angle, $\theta$. While for a dc current of $I=6 \mathrm{~mA}$ a sharp change between $0^{\circ}$ (parallel alignment) and $180^{\circ}$ (antiparallel alignment) is revealed, for higher dc current values of $I_{\mathrm{dc}}=7.5$ and $9 \mathrm{~mA}$ a narrow field region exists for which the stable values of $\theta$, in between the parallel and antiparallel orientation, are allowed corresponding to the steady-state precession. The plots together with the phase diagram also show that for $7<I_{\mathrm{dc}}<12 \mathrm{~mA}$ the precession can be excited only when the magnetic field is starting from negative values [solid curves in Figs. 6(b)-6(d)]. In contrast, above $12 \mathrm{~mA}$ the precession can be obtained for both sweep directions of the applied field.

Finally, we would like to make a remark on thermal fluctuations. They have a noticeable influence on the critical currents of the phase diagram moving the boundaries of the hysteretic and nonhysteretic regions, so that such limits acquire a degree of uncertainty. ${ }^{27}$ The main effects are that thermal fluctuations cause transitions between magnetic states and, therefore, the width of the hysteretic region depends on temperature. ${ }^{27}$ Finite-temperature effects can be studied through statistical descriptions or by adding a random field to the effective field, thus changing the theoretical analysis from a deterministic study to a statistical one. ${ }^{27}$ Such effects were ignored here.

\section{CONCLUSION}

In summary, we have shown that mircowave irradiation with a frequency close to the eigenprecession frequency affects the spin-transfer-torque-driven motion of the magnetization. This was performed on perpendicularly magnetized nanopillars for which no conventional experimental method allows a determination of the precession frequency. Furthermore, it was shown that the critical current density can be strongly reduced by microwave irradiation. The method provides a powerful tool to study magnetization dynamics not only in nanopillar spin valves with perpendicular anisotropy but also, in general, in nanostructured samples. The results of the measurements were compared to micromagnetic and macrospin calculations. They revealed that the modes observed in the resistance measurements, indeed, stem from a steady-state precession of the spins in the sample. In addition to the eigenresonance, magnetostatic forward-volume modes are excited.

\section{ACKNOWLEDGMENTS}

We acknowledge financial support by the Deutsche Forschungsgemeinschaft (SFB 491) and National Science Foundation Award No. DMR-1008654, CONICYT, FONDECYT 11080246, the program "Financiamiento Basal para Centros Científicos y Tecnológicos de Excelencia" CEDENNA FB0807, ISTRADE, and FRIENDS ANR program.

\section{APPENDIX A: MICROWAVE GENERATION}

To enable microwave broadband irradiation, a coaxial semirigid microwave cable (SRMC) with a diameter of $2 \mathrm{~mm}$ is utilized. To produce a high-frequency magnetic field in the film plane and perpendicular to the external field, the SRMC is electrically shorted at its end by connecting the inner conductor and the ground shield of the SRMC. The direction of the short is oriented perpendicular to the external magnetic field, $B$. The electric field of the microwave induces a current along the short, which results in a magnetic field around the short and perpendicular to $B$, as schematically shown in Fig. 7(a). While the shorted SRMC can be employed also to detect FMR by measuring the reflected microwave power, ${ }^{28}$ in our case it is used for excitation only. The performance of the shorted 

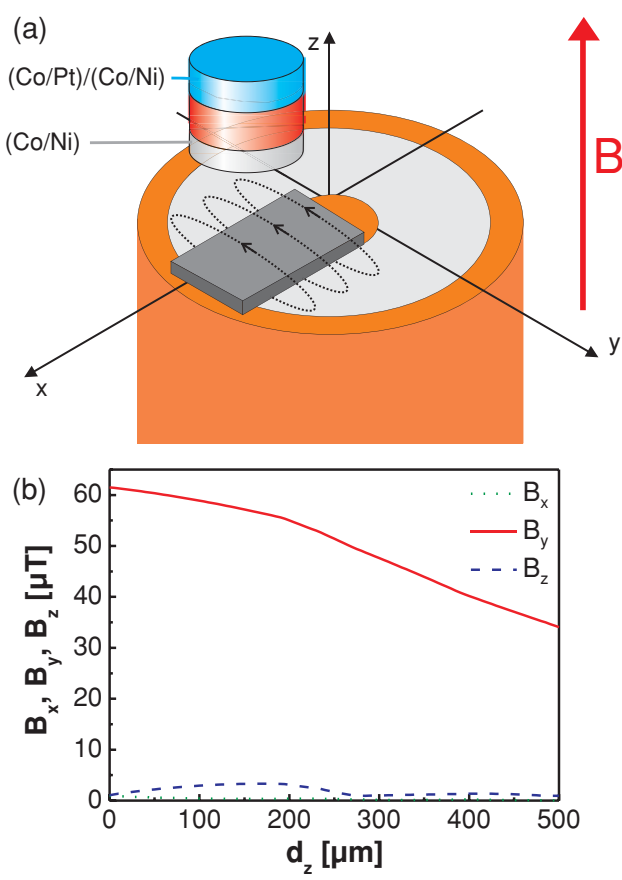

FIG. 7. (Color online) (a) Sketch of a coaxial semirigid microwave cable with generated microwave field and sample. (b) Dependence of the $x, y$, and $z$ components of the microwave field on the distance from the short in the middle of the short platelet.

SRMC has been simulated using the finite-element-method simulation software HFSS (by Ansoft). Figure 7(b) shows the simulation of all components of the high-frequency magnetic field with respect to the coordinate system from Fig. 7(a) as a function of the distance between short and sample. While the $x$ and $z$ components are almost zero, there is a strong $y$ component perpendicular to the rf current direction ( $y$ direction) in the short. This field drives the magnetization. Due to the broadband properties of the SRMC, the frequency of this excitation field can be varied from about 6 to $18 \mathrm{GHz}$. The shape of the short, chosen to be a thin platelet, strongly influences the $1 / r$ decay as a function of the distance to the sample, expected for a short with circular cross section and radius $r$. In the case of the platelet, the decrease is far less pronounced and even at a distance of about $0.5 \mathrm{~mm}$ the field amplitude still exhibits about half of its value at the surface of the short. The typical sample distance is $0.25-0.5 \mathrm{~mm}$. In order to increase the signal strength of the microwave generator, we use an amplifier with a maximal output power of $1 \mathrm{~W}$.

\section{APPENDIX B: CALCULATION OF CRITICAL REGIONS IN THE PHASE DIAGRAM}

By inserting the expressions for $\dot{m}_{x}$ and $\dot{m}_{y}$, given by Eq. (3), into the equation for $\dot{m}_{z}$, and taking into account that $\vec{m} \cdot \dot{\vec{m}}=0$, we obtain the following equation:

$$
\begin{aligned}
& \dot{m}_{z}\left[1-\alpha^{2}\left(m_{z}^{2}-1\right)\right]=\alpha^{2} m_{z}\left(m_{x} \dot{m}_{x}+m_{y} \dot{m}_{y}\right) \\
& -\gamma\left(m_{z}^{2}-1\right)\left[\beta+\alpha\left(B+M_{\mathrm{eff}} m_{z}\right)\right] .
\end{aligned}
$$

By evaluating the derivatives

$$
\frac{d}{d t}\left(m_{x}^{2}+m_{y}^{2}\right)=2\left(m_{x} \dot{m}_{x}+m_{y} \dot{m}_{y}\right)
$$

and

$$
\frac{d}{d t}\left(m_{x}^{2}+m_{y}^{2}\right)=\frac{d}{d t}\left(1-m_{z}^{2}\right)=-2 m_{z} \dot{m}_{z}
$$

it follows that $m_{x} \dot{m}_{x}+m_{y} \dot{m}_{y}=-m_{z} \dot{m}_{z}$. In this case, Eq. (4)can be written as

$$
\begin{aligned}
\dot{m}_{z}\left[1-\alpha^{2}\left(m_{z}^{2}-1\right)\right]= & -\alpha^{2} m_{z}^{2} \dot{m}_{z} \\
& -\gamma\left(m_{z}^{2}-1\right)\left[\beta+\alpha\left(B+M_{\mathrm{eff}} m_{z}\right)\right]
\end{aligned}
$$

or

$$
\dot{m}_{z}\left(1+\alpha^{2}\right)=-\gamma\left(m_{z}^{2}-1\right)\left[\beta+\alpha\left(B+M_{\mathrm{eff}} m_{z}\right)\right] .
$$

This yields the following equation for $\dot{m}_{z}$ :

$$
\dot{m}_{z}=\frac{\gamma\left(1-m_{z}^{2}\right)\left[\beta+\alpha\left(B+M_{\mathrm{eff}} m_{z}\right)\right]}{1+\alpha^{2}} .
$$

From this equation we can extract the static solutions for $d\left(m_{z}\right) / d t=0$. These are $m_{z}= \pm 1$ as well as the nontrivial solution given by $\beta p_{z}+\alpha\left(B+M_{\text {eff }} m_{z}\right)$. From the former equation we can obtain the critical currents that separate regions in the $B-I_{\mathrm{dc}}$ phase diagram, where the parallel or antiparallel states lose their stability,

$$
\begin{gathered}
I_{\mathrm{dc}}^{\mathrm{P}}=-\frac{2|e| M_{S} V \alpha}{h g_{P}(1)}\left(B+M_{\mathrm{eff}}\right), \\
I_{\mathrm{dc}}^{\mathrm{AP}}=-\frac{2|e| M_{S} V \alpha}{h g_{P}(-1)}\left(B-M_{\mathrm{eff}}\right) .
\end{gathered}
$$

In fact, the same critical regions were obtained by Mangin $\mathrm{et}$ al. [see Eqs. (2a) and (2b) in Ref. 15]. The critical currents are shown in Fig. 6(a), where $I_{\mathrm{dc}}^{\mathrm{P}}$ is represented by the blue line and $I_{\mathrm{dc}}^{\mathrm{AP}}$ by the yellow line. Note that these curves have different slopes due to the dependence on the spin-torque polarization factor, $g_{P}$, on the magnetization. This fact ensures that both curves cross at a critical point $\left(B^{*}, I_{\mathrm{dc}}^{*}\right)$, where $I_{\mathrm{dc}}^{\mathrm{P}}=I_{\mathrm{dc}}^{\mathrm{AP}}$, above which there is no hysteretic behavior. The critical field and current are given by

$$
\begin{aligned}
& B^{*}=-M_{\mathrm{eff}} \frac{g_{p}(-1)+g_{p}(1)}{g_{p}(-1)-g_{p}(1)}, \\
& I_{\mathrm{dc}}^{*}=-\frac{4|e| M_{S} V \alpha M_{\mathrm{eff}}}{\left[g_{p}(-1)-g_{p}(1)\right]} .
\end{aligned}
$$

Then, for the currents above this threshold, precessional states should take place in a small field window, which increases with a current. The precession is characterized by a stable value of $m_{z}$ that must be a solution of

$$
m_{z}=-\frac{B}{M_{\mathrm{eff}}}-\frac{h g_{p}\left(m_{z}\right) I_{\mathrm{dc}}}{2|e| M_{S} V \alpha M_{\mathrm{eff}}}
$$

or

$$
m_{z}=-\frac{B}{M_{\mathrm{eff}}}-\frac{2 g_{p}\left(m_{z}\right)}{\left(g_{p}(-1)-g_{p}(1)\right)} \frac{I_{\mathrm{dc}}}{I_{\mathrm{dc}}^{*}} .
$$

One should note that this solution is valid only for currents above $I_{\mathrm{dc}}^{*}$ and for fields in the precessional region. 
*nathalie.reckers@uni-due.de

${ }^{1}$ L. Berger, Phys. Rev. B 54, 9353 (1996).

${ }^{2}$ J. Slonczewski, J. Magn. Magn. Mater. 159, L1 (1996).

${ }^{3}$ J. A. Katine, F. J. Albert, R. A. Buhrman, E. B. Myers, and

D. C. Ralph, Phys. Rev. Lett. 84, 3149 (2000).

${ }^{4}$ E. B. Myers, D. C. Ralph, J. A. Katine, R. N. Louie, and

R. A. Buhrman, Science 285, 867 (1999).

${ }^{5}$ O. Boulle, V. Cros, J. Grollier, L. G. Pereira, C. Deranlot, F. Petroff, G. Faini, J. Barnas, and A. Fert, Nat. Phys. 3, 492 (2007).

${ }^{6}$ S. I. Kiselev, J. C. Sankey, I. N. Krivorotov, N. C. Emley, R. J. Schoelkopf, R. A. Buhrman, and D. C. Ralph, Nature (London) 425, 380 (2003).

${ }^{7}$ A. Slavin and V. Tiberkevich, IEEE Trans. Magn. 45, 1875 (2009).

${ }^{8}$ S. H. Florez, J. A. Katine, M. Carey, L. Folks, and B. D. Terris, J. Appl. Phys. 103, 07A708 (2008).

${ }^{9}$ M. Tsoi, A. G. M. Jansen, J. Bass, W.-C. Chiang, V. Tsoi, and P. Wyder, Nature (London) 406, 46 (2000).

${ }^{10}$ S. Mangin, Y. Henry, D. Ravelonsona, J. A. Katine, and E. E. Fullerton, Appl. Phys. Lett. 94, 012502 (2009).

${ }^{11}$ D. Houssameddine, U. Ebels, B. Delaet, B. Rodmacq, I. Firastrau, F. Ponthenier, M. Brunet, C. Thirion, J.-P. Michel, L. Prejbeanu-Buda, M.-C. Cytrille, O. Redon, and B. Dieny, Nat. Mater. 6, 447 (2007).

${ }^{12}$ W. Chen, J.-M. L. Beaujour, G. de Loubens, J. Z. Sun, and A. D. Kent, Appl. Phys. Lett. 92, 012507 (2008).

${ }^{13}$ M. Carpentieri, G. Finocchio, B. Azzerboni, and L. Torres, Phys. Rev. B 82, 094434 (2010).

${ }^{14}$ M. C. McGregor, J. Sci. Instrum. 43, 825 (1966).

${ }^{15}$ S. Mangin, D. Ravelonsona, J. A. Katine, M. J. Carey, B. D. Terris, and E. E. Fullerton, Nat. Mater. 5, 210 (2006).
${ }^{16}$ S. Mangin, D. Ravelonsana, Y. Henry, J. A. Katine, and E. E. Fullerton, Assoc. Asia Pac. Phys. Soc. Bull. 18, 6 (2008).

${ }^{17}$ D. Ravelosona, S. Mangin, Y. Lemaho, J. A. Katine, B. D. Terris, and E. E. Fullerton, Phys. Rev. Lett. 96, 186604 (2006).

${ }^{18}$ A. Fukushima, H. Kubota, A. Yamamoto, Y. Suzuki, and S. Yuasa, J. Appl. Phys. 99, 08H706 (2006).

${ }^{19}$ D. Ravelosona, S. Mangin, Y. Henry, Y. Lemaho, J. A. Katine, B. D. Terris, and E. E. Fullerton, J. Phys. D 40, 1253 (2007).

${ }^{20}$ S. Kaka, M. R. Pufall, W. H. Rippard, T. J. Silva, S. E. Russek, and J. A. Katine, Nature (London) 437, 389 (2005).

${ }^{21}$ F. B. Mancoff, N. D. Rizzo, B. N. Engel, and S. Tehrani, Nature (London) 437, 393 (2005).

${ }^{22}$ [http://math.nist.gov/oommf/].

${ }^{23}$ S. O. Demokritov and B. Hillebrands, in Spin Dynamics in Confined Magnetic Structures I, Topics in Applied Physics, edited by B. Hillebrands and K. Ounadjela, Vol. 83 (Springer Verlag, Berlin, 2003), p. 65 .

${ }^{24}$ O. Posth, C. Hassel, M. Spasova, S. Mangin, G. Dumpich, and J. Lindner, J. Appl. Phys. 106, 023919 (2009).

${ }^{25}$ M. Beleggia, M. De Graef, Y. T. Millev, D. A. Goode, and G. Rowlands, J. Phys. D 38, 3333 (2005).

${ }^{26}$ R. Lehndorff, D. E. Bürgler, A. Kakay, R. Hertel, and C. M. Schneider, IEEE Trans. Magn. 44, 1951 (2008).

${ }^{27}$ M. D. Stiles and J. Miltat, in Spin dynamics in Confined Magnetic Structures III, Spin Transfer Torque and Dynamics, edited by B. Hillebrands and A. Thiaville, Vol. 101 (Springer Verlag, Berlin, Heidelberg, 2006), p. 225.

${ }^{28} \mathrm{~F}$. M. Römer et al. (to be published). 